\title{
Endometrioma suburetral
}

\author{
Cabral Ribeiro J, Pérez García D, Martins Silva C, Ribeiro Santos A. \\ Servicio de Urología. Hospital de São Marcos. Braga, Portugal.
}

Actas Urol Esp. 2007;31(2):153-156

\section{RESUMEN}

\section{ENDOMETRIOMA SUBURETRAL}

La endometriosis es una enfermedad común que afecta con preferencia a mujeres en edad fértil. Están implicados sobre todo órganos pélvicos internos y de forma ocasional puede tener localizaciones raras. Describimos el caso clínico de una paciente de 32 años de edad con dispareunia, disuria, dolor uretral y masa dolorosa suburetral que condicionaba sintomatología miccional obstructiva. El diagnóstico de sospecha era de un divertículo de uretra complicado o de un endometrioma suburetral. La exéresis quirúrgica realizada confirmó tratarse de un quiste de endometriosis. Se realizan breves comentarios sobre esta localización rara de endometriosis y sus implicaciones en el diagnóstico diferencial con otras entidades quísticas vaginales.

Palabras clave: Endometriosis. Divertículo uretral. Quiste vaginal.

\section{ABSTRACT \\ SUBURETHRAL ENDOMETRIOMA}

Endometriosis is a common disease affecting mostly women in childbearing age. It usually involves the internal pelvic organs but occasionally can present in unusual locations.

We report on a 32-year-old nulliparos female presenting with a suburethral painful mass causing obstructive voiding symptoms; she had also a long history of dismenorreia, perimenstrual pelvic pain, urinary tract infections, disúria and dispareunia. The diagnostic work-up revealed a suburethral cystic lesion consistent with a complicated urethral diverticulum or a suburethral endometrioma. Complete surgical excision confirmed an endometriotic cyst.

The differential diagnosis of this rare suburethral location of endometriosis with other vaginal cysts is discussed.

Keywords: Endometriosis. Urethral diverticulum. Vaginal cysts.

$\mathrm{L}^{2}$ a endometriosis es una enfermedad frecuente afectando a 3-10\% de las mujeres en edad fértil. Siendo aun más prevalente en la consulta de infertilidad (25-35\%) ${ }^{1}$. Se define como la existencia de tejido endometrial funcionante fuera de la cavidad uterina. La localización de la endometriosis es fundamentalmente pélvica (útero, trompa de Falopio, ovarios, ligamentos úterosagrados, peritoneo pélvico y fondo de saco). La localización extrapélvica no es rara: Existen casos descritos en múltiples áreas del cuerpo, incluyendo la cavidad torácica y pulmón, aparato gastrointestinal, la pared abdominal y extremidades, incisiones quirúrgicas, vagina y tracto urinario ${ }^{2}$.
En cerca de 1 a $2 \%$ de los casos de endometriosis envuelve el tracto urinario más frecuente la vejiga, seguida por el ureter, riñón y raramente la uretra ${ }^{3}$.

Presentamos un caso raro de un foco de endometriosis de la localización suburetral causante de sintomas miccionales obstructivos.

\section{CASO CLÍnICo}

Paciente de sexo femenino de 32 años, nulípara, sin antecedentes quirúrgicos, fue observada por masa a nivel de la pared vaginal anterior que condicionaba dificultad miccional. 
Presentaba una larga historia de dismenorrea asociada con irregularidades menstruales hasta hace cerca de 5 años, momento en que inicio anticonceptivos orales debido al inicio de la actividad sexual. Hace dos años inició de forma progresiva sintomatología de disuria, dolor pélvica y dispareunia con agravamiento en el período menstrual asociada a ocasionales infecciones urinarias. Un año después notó una masa elástica y dolorosa que se insinuaba a nivel del vestíbulo vaginal. El estudio realizado reveló una lesión quística que fue sometida a punción aspirativa con salida de contenido hemático espeso. El estudio citológico no fue concluyente. Después de una mejoría transitoria e inicial de la sintomatología que duró algunos meses, se verificó un agravamiento del dolor pélvico perimenstrual, de la dismenorrea, del dolor uretral y disuria. La masa vaginal anterior aumentó de dimensiones y por sufrir protusión vaginal pasó a condicionar dificultad creciente en el inicio de la micción con esfuerzo miccional aumentado, fuerza de chorro disminuido, intermitente con necesidad de reducción digital por la paciente al iniciar la micción. En la exploración objetiva, presentaba una masa elástica, muy dolorosa, bien delimitada, de unos 3-4 cm, localizada en la pared vaginal anterior y que se insinuaba en el introito vaginal.
La RMN pélvica identificó una lesión redondeada, bien delimitada, localizada posteriormente a la uretra de 3,9x2,9x3 cm., con hiperseñal en $\mathrm{T} 1$ y $\mathrm{T} 2$ y que se mantiene en las imágenes de saturación de grasa, aspecto que sugiere lesión quística con contenido proteico ó de tipo hemorrágico. Las sospechas diagnósticas son de un divertículo de uretra complicado de infección y hemorragia o un endometrioma (Fig. 1).

La urografía endovenosa reveló normalidad excretora sin evidencia de ectopia o duplicidad ureteral. Apenas se identifica una imagen regular de substracción a nivel del pavimento vesical. La uretrografía no documentó la presencia de ninguna comunicación con la uretra. Fue sometida a uretrocistoscopia con anestesia, no reveladora de ostio diverticular y en seguida fue efectuada una resección de la masa por vía transvaginal (Fig. 2). Se resecó la totalidad de la pared que se extendía hasta el nivel del cuello vesical y no fue documentada ninguna comunicación con la uretra. La lesión contenía un líquido hemático oscuro y espeso (tipo chocolate). El examen anatomopatológico demostró endometriosis (Fig. 3). En el postoperatorio inmediato tuvo un período transitorio de ligera incontinencia urinaria (asociada a urgencia y a esfuerzos moderados) que cedió con tratamiento anticolinérgico y ejercicios pélvicos.
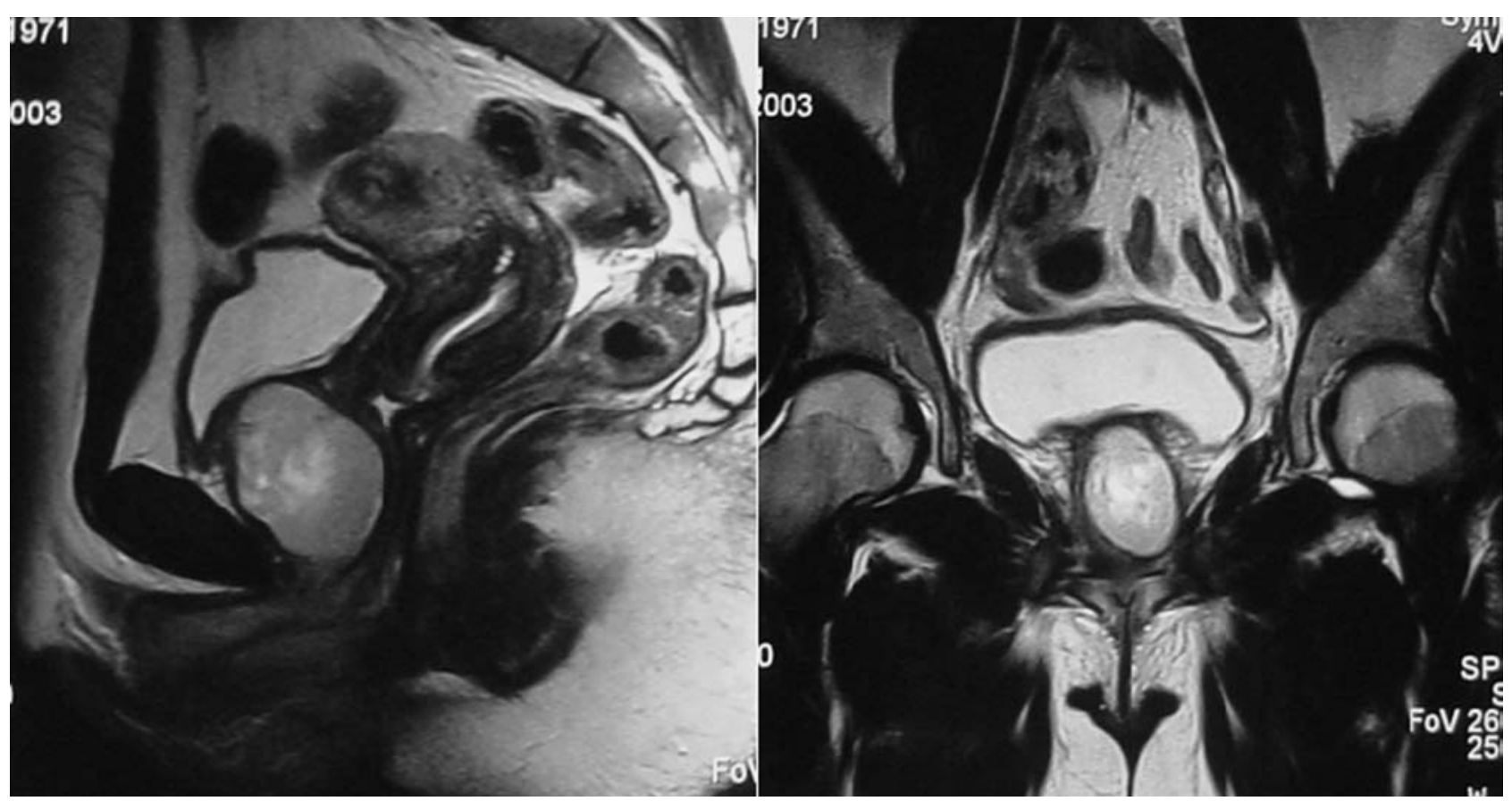

FIGURA 1. RMN 


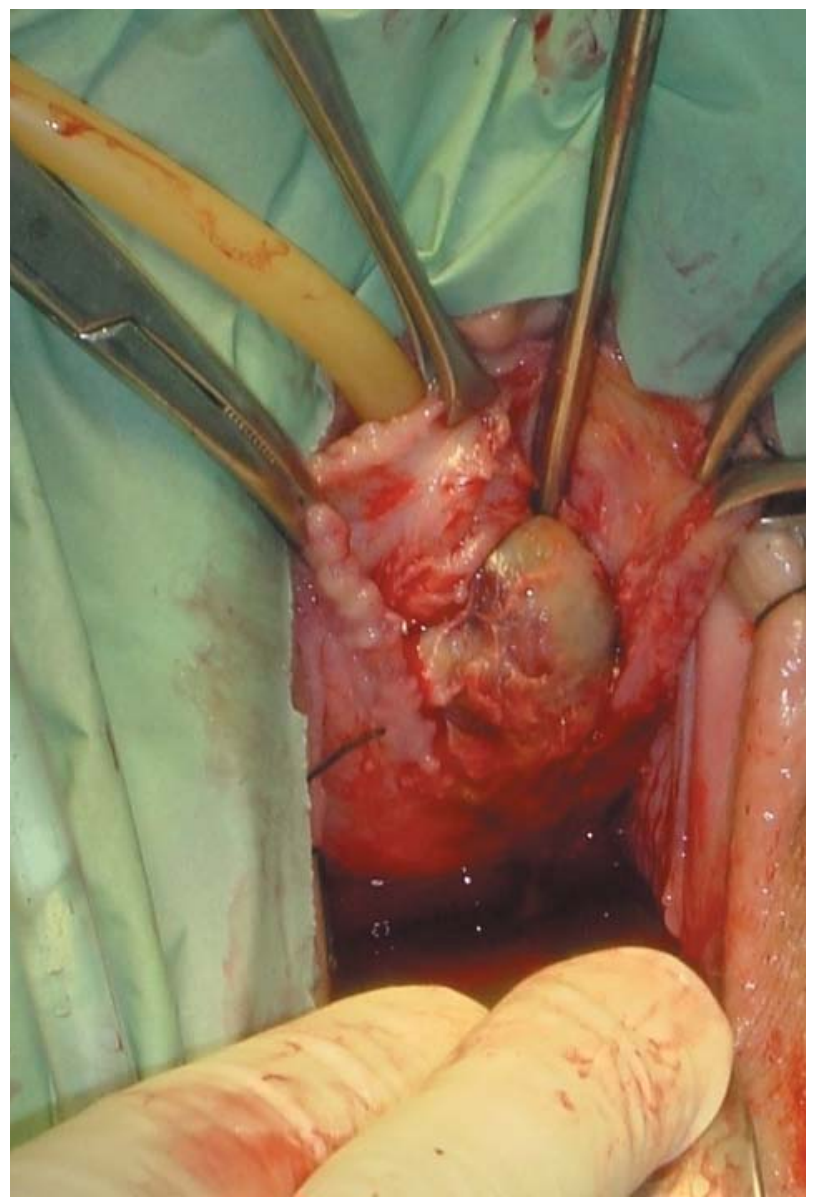

FIGURA 2. Imagen intraoperatória.

Realizó tratamiento adyuvante durante 6 meses con triptorelina 3,75 mg mensual: Actualmente se encuentra asintomática y sin evidencia de recidiva.

\section{COMENTARIOS}

La endometriosis fue descrita por primera vez por Von Rokitansky en 1860 y se caracteriza histológicamente por la presencia de glándulas endometriales y/o estroma y aglomerados de macrófagos repletos de pigmento hemosidérico en localización extra uterina ${ }^{1}$.

Aunque pueda ser diagnostica en cualquier edad, se detectan en la mayoría de los casos en una mujer en edad fértil (20-45 años). Menos de $5 \%$ de los casos afectan a mujeres después da la menopausia. $^{1}$

La endometriosis es una enfermedad benigna ya que le endometrio, incluso ectópico, es incapaz de crecimiento autónomo. Puede sin embargo pre-

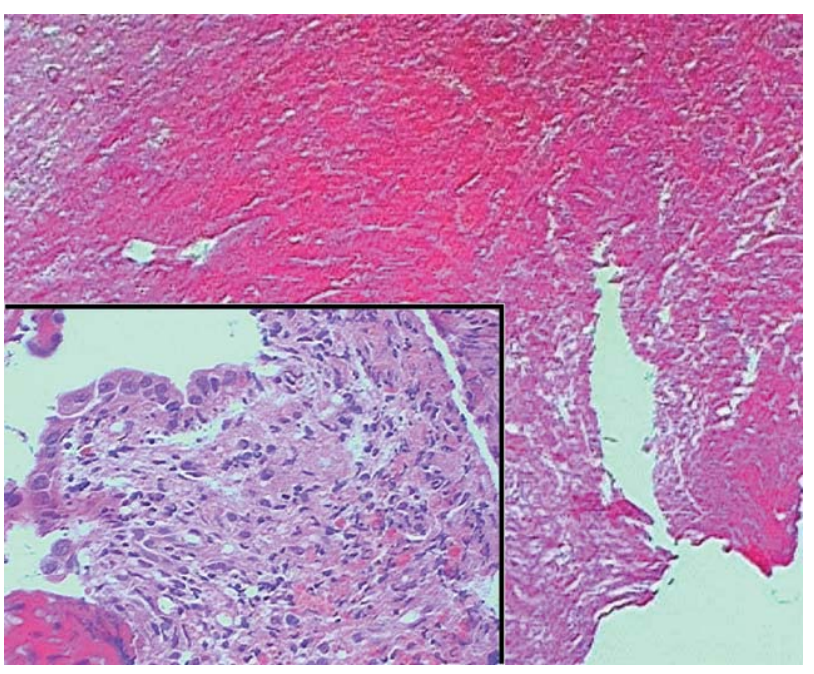

FIGURA 3. Pared de la lesión con áreas de hemorragia intersticial (HE 40X). Epitelio de revestimiento cúbico simple $e$ infiltrado inflamatorio de histiocitos con contenido de hemosiderina - endometriosis (caja-HE40OX).

sentar un comportamiento agresivo y sintomático debido a la intensa reacción inflamatoria y fibrótica que condiciona, provocando por ejemplo obstrucciones ureterales ${ }^{4}$.

La localización de los focos de endometriosis es sobre todo pélvica, pero la enfermedad extrapélvica no es rara. El tracto urinario está afectado en cerca de 1 a $2 \%$ de los casos de endometriosis. La localización vesical (80-85\%) seguida por la ureteral, renal y raramente la uretra (ratio 40:7:2:1) ${ }^{5}$.

La endometriosis vaginal es sobre todo intrínseca con lesiones fácilmente observables con espéculo, o cuando envuelve la pared tiene a formar endometriomas a nivel del fondo vaginal y septo rectovaginal ${ }^{6}$.

La localización suburetral es excepcional encontrándose apenas 3 casos adicionales descritos en la literatura, uno de ellos en una mujer post menopáusica ${ }^{7-9}$.

La presentación clínica de estos casos no difiere en términos sintomáticos de la presentación de las diversas entidades quísticas que pueden envolver a vagina y a la uretra. Así, síntomas con larga evolución de dispareunia, disuria, dolor pélvico, infecciones urinarias, dificultades miccionales, asociadas a una lesión quística vaginal anterior implican el diagnóstico diferencial con alteraciones quísticas de origen embrionario de la pared vaginal (quistes de Gardner ó de Muller), quistes de las glándulas de Skene y de Bartolin, la adenosis vaginal, divertículos uretrales y la endometriosis ${ }^{6}$. 
Síntomas más específicos como el goteo post miccional, más típico de la enfermedad diverticular, las irregularidades menstruales, la dismenorrea, la infertilidad y el típico agravamiento sintomático perimenstrual más indicativos de la endometriosis, pueden auxiliar en este esfuerzo diagnóstico. El examen de elección para la valoración de la uretra es la RMN que presenta excelente sensibilidad para el diagnóstico tanto del divertículo de uretra como de la endometriosis pélvi$\mathrm{ca}^{10,11}$. No obstante, la sobreposición de hiperseñal, principalmente en divertículos con infección, puede colocar dificultades de diagnóstico diferencial. Pero el diagnóstico definitivo pasa por la exéresis quirúrgica o biopsia de la lesión. El tratamiento de la endometriosis depende de la edad de la mujer, de la voluntad de mantener la fertilidad, de la extensión y localización de los focos de enfermedad, del grado de alteración menstrual, del impacto sexual y de la coexistencia de otra afectación pélvica ${ }^{1,3,12}$.

Las terapéuticas varían así desde una actitud expectante (en los casos asintomáticos), un tratamiento médico de manipulación hormonal o quirúrgico (exéresis o destrucción aislada de lesiones, o radical con histerectomía asociada a ooforectomía bilateral) ${ }^{12}$.

El endometrio es un tejido hormosensible expresando receptores estrogénicos, progestagénicos y androgénicos. Este tejido incluso en localización ectópica, presenta las 3 fases del ciclo endometrial; proliferación, secreción e fase decidual. Es típicamente en la fase decidual, en que la hemorragia y necrosis celular condicionan un aumento del volumen del endometrioma y el agravamiento del proceso inflamatorio y fibrótico local, que corresponde a un período de exacerbación sintomática.

Varios esquemas terapéuticos de manipulación hormonal tienen sido aplicados. Anticonceptivos orales compuestos de estrógeno y progestagéneo, el danazol o agonistas LHRH tienen sido utilizados para condicionar un estado de pseudo menopausia $^{12}$.

Sin embargo el tratamiento de elección para las localizaciones suburetrales, así como para endometriomas (quistes de dimensiones superiores a $2 \mathrm{~cm}$ ) es una exéresis quirúrgica ${ }^{12}$. Esta permite simultáneamente el tratamiento y el diagnóstico histológico.
En los casos descritos de divertículos de uretra voluminosos con extensión hasta el cuello vesical y retrotrigonales, existe un riesgo elevado de incontinencia postoperatoria. Esta puede prevenir-se (en los casos de incontinencia previa) o corregida posteriormente por la colocación de un sling suburetral ${ }^{13-14}$. Nuestra paciente refirió transitoriamente ligera incontinencia urinaria asociada a síntomas de inestabilidad vesical y que resolvió solo con terapéutica conservadora.

\section{CONCLUSIÓN}

Aunque sea rara, la endometriosis debe ser considerada en la valoración diagnóstica de cualquier masa quística periuretral, especialmente si se asocia a historia de dolor pélvica, dispareunia, dismenorrea (o alteraciones menstruales) y dificultad miccional.

\section{REFERENCIAS}

1. Bologna RA, Whitmore K. Genitourinary endometriosis. AUA Update series 2000; 32-37.

2. Markham SM, Carpenter SE, Rock JA. Extrapelvic endometriosis Obstet Gynecol Clin North Am. 1989;16(1):193-219.

3. Comiter CV. Endometriosis of the urinary tract. Urol Clin N Am. 2002;29(3):625-635.

4. Acker O, Robert Y, Carpentir F, Vinatier D, Cosson M. Endométriose vésicale ou urétérale, symptomatique: à propos de 8 cas et revue de la litérature. Ann Chir. 2003;128(1):34-39.

5. Vesga Molina F, Serrano Bartolome JM, Gutierrez Minguez E, Tello Royloa A, Echevarria Iturbe C, Marcos Diaz J. Endometriosis ureteral. Arch Esp Urol. 2000;53(5):470-472.

6. Eilber KS, Raz S. Benign cystic lesions of the vagina: A literature review. J Urol. 2003;170(3):717-722.

7. Wu YC, Liang CC, Soong YK. Suburethral endometrioma. A case report. J Reprod Med. 2003;48(3):204-205.

8. Rabinerson D, Avrech O, Kaplan B, BraslaVsky D, Goldman GA, Neri A. Endometrioma of the vagina in menopause. Acta Obstet Gynecol Scand. 1996;75(5):506-507.

9. Chowdhry AA, Miller FH, Hammer RA. Endometriosis presenting as a urethral diverticulum. A case report. J Reprod Med. 2004;49(4):321-323.

10. Arzoz Fabregas M, Ibarz Servio L, Areal Calama J, Saladie Roig JM. Divertículos de uretra femenina. Arch Esp Urol. 2004,57(4):381-388.

11. Zawin M, McCarthy S, Scout L, Comite F. Endometriosis: Appearance and detection at MR imaging. Radiology. 1989;171(3):693-696.

12. Frackiewicz EJ. Endometriosis: An overview of the disease and its treatment. J Am Pharm Assoc. 2000;40(5):645-657; quiz 699-702.

13. Romanzi LJ, Groutz A, Blaivas JG, Urethral diverticulum in women: Diverse presentations resulting in diagnostic delay and mismanagement. J Urol. 2000;164(2):428-433.

14. Aspera AM, Rackley RR, Vasavada SP. Contemporary evaluation and management of the female urethral diverticulum. Urol Clin North Am. 2002;29(3):617-624.

Correspondencia autor: Dr. J. Cabral Ribeiro Servicio de Urología. Hospital de São Marcos Apartado 2242. 4701-965 Braga (Portugal). Telf: +351253209110 E-mail autor: jcabral@hsmbraga.min-saude.pt Información artículo: Original - Nota clínica

Trabajo recibido: noviembre 2005

Trabajo aceptado: abril 2006 